\title{
A research of heat transfer enhancement of rectangular channel with dimples
}

\author{
Zhe Fu' ${ }^{\mathrm{a}}$, Yingping Qian ${ }^{\mathrm{b}}$, Xizhi Zhou ${ }^{\mathrm{c}}$, Zhihong Yan ${ }^{\mathrm{d}}$ and Yang Jiao ${ }^{\mathrm{e}}$ \\ College of Mechanical Engineering of Hubei University of Technology, Hongshan District, Wuhan City, Hubei Province, PRC

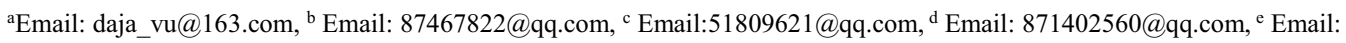 \\ 2449558169@qq.com
}

\begin{abstract}
: this thesis is aimed to do numerical simulations on rectangular channel with a new model of dimple in different condition of channel height, dimple depth, dimple distance by using CFD program and RNG k- $\varepsilon$ model. It is concluded that, the existence of dimple has changed the velocity and direction of the fluid, as well as the original flow status. Dimple increased the heat exchange area of rectangular channel and broke the boundary layer, enhanced the flow rate of fluid, as a result of enhanced heat transfer performance.
\end{abstract}

Keywords: dimple, heat transfer enhancement, heat transfer performance, numerical simulation.

\section{Introduction}

In recently technology of heat transfer enhancement, in order to enhance the ability of heat exchange in fluid, most employers use the methods of roughen layer surface and extend the surface area. The general method is using horizontal or spiral fin, thread, groove and dimple to enhance heat transfer [1,2,3]. As for the research of heat transfer enhancement, not only need to take enhancement performance in to consideration but also have to consider the friction factors of fluid. The heat transfer enhancement of dimple breaks the boundary layer because of the sunken surface, and creates different sizes of vortex which accelerates the heat exchange in rectangular channel.

The factors of heat exchange and flow friction in dimple heat transfer enhancement are mainly affected by the shape, depth, distance, Re of dimple. Burgess [4] used air as medium and analyzed the effect of dimple depth, discovered that heat exchange factor $\eta$ and friction factor are increased by the depth went up. Mahmood [5] used Infrared Thermometric Techniques to find out that leeward side of dimple has weaker heat exchange than windward side. Chen. J [6] has done the analysis of how the distance and depth and concentration affect heat transfer, ending up with found a best way to design heat transfer enhancement. Bunker [7] found that compared dimple to smooth channel, dimple has twice heat enhancement and 2 7 times stronger friction. Somin[8] has a research on channel height and dimple queue mode when Re between 30000 50000, found that heat transfer performance is good with high concentration dimple when the channel height kept constantly, and with narrow rectangular channel in the same dimple queue mode. The author analyzed that in different Re, the effect of channel height, dimple depth, dimple distance on heat transfer and friction in a new dimple and after that analyzed the integrated heat transfer performance under different individual working conditions.

\section{Models and Judge Standard}




\subsection{Physical Model}

The Figure 1 below is the construction and geometry factors of dimple heat transfer rectangular channel. Channel height is $\mathrm{H}$, dimple depth is $\mathrm{H}_{\mathrm{d}}$, dimple distance is $\mathrm{S}$, this thesis has done numerical simulation of models: $\mathrm{H}=2 \mathrm{~mm} 、 4 \mathrm{~mm}$. $6 \mathrm{~mm} 、 8 \mathrm{~mm} ; \mathrm{H}_{\mathrm{d}}=0.4 \mathrm{~mm} 、 0.8 \mathrm{~mm} 、 1.3 \mathrm{~mm} 、 1.6 \mathrm{~mm} ; \mathrm{S}=4 \mathrm{~mm} 、 9 \mathrm{~mm} 、 14 \mathrm{~mm} 、 18 \mathrm{~mm}$, in $\mathrm{Re}=2000,4000,7000$, 10000 , take rectangular dimple which length: width $=2: 1$, vertical distance: horizontal distance $=1: 1.3$.

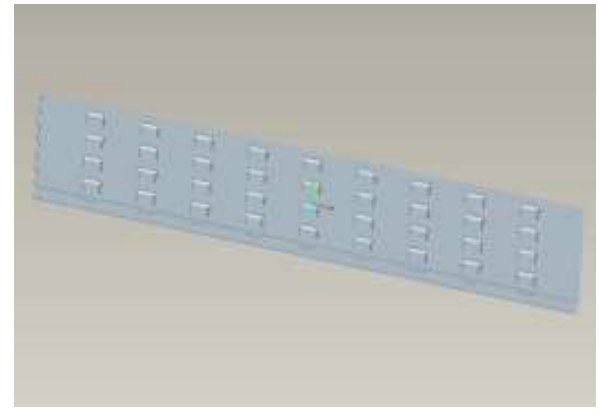

(a) Construction of dimple boa

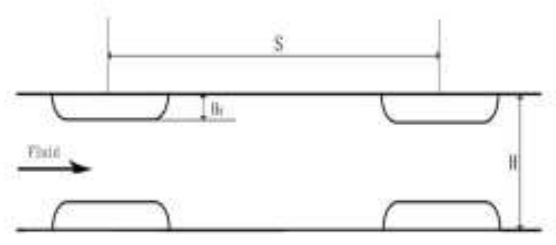

(b) Geometry factors of rectangular channel

Fig. 1. Physical model of rectangular channel.

\subsection{Mathematic Model and Formula}

Continuity equation, momentum equation, energy equation of fluid can be all presented as general equation:

$$
\begin{aligned}
& \frac{\partial(\rho u \varphi)}{\partial x}+\frac{\partial(\rho v \varphi)}{\partial y}+\frac{\partial(\rho w \varphi)}{\partial z}= \\
& \frac{\partial}{\partial x}\left(\Gamma_{\varphi, t} \frac{\partial \varphi}{\partial x}\right)+\frac{\partial}{\partial y}\left(\Gamma_{\varphi, t} \frac{\partial \varphi}{\partial y}\right)+\frac{\partial}{\partial z}\left(\Gamma_{\varphi, t} \frac{\partial \varphi}{\partial z}\right)+S_{\varphi}
\end{aligned}
$$

The heat transfer performance, friction performance and integrated heat transfer performance of dimple board are presented as $\mathrm{Nu}, \mathrm{f}, \mathrm{\eta}$. In the experiments, the $\mathrm{Nu} 0$ and $\mathrm{f} 0$ are calculated by numerical simulation. The $\mathrm{Re}, \mathrm{Nu}, \mathrm{f}, \eta$ are defined as:

$$
\begin{array}{r}
R_{e}=\frac{D_{H} \rho U}{\mu} \\
N_{u}=\frac{q D_{H}}{\left(T_{\text {wall }}-T_{\text {ref }}\right) \lambda} \\
f=\frac{2 \Delta P D_{H}}{\rho U^{2}} \\
\eta=\frac{N_{u} / N_{u_{0}}}{\left(f / f_{0}\right)^{1 / 3}}
\end{array}
$$

According to different Re convert into fluid mass Qm, and set Qm as initial value, both sides are meristic boundary, fluid in channel is air, fluid temperature is $600 \mathrm{~K}$, all calculation are carried out by SIMPLE and RNG k-ع. Momentum, Turbulent kinetic energy, Turbulent fluctuation dissipation rate and energy equation are second-order upwind scheme. The convergence precision of continuity equation is 10-6, ignore fouling resistance and gravity, phase transfer and reacting flow are not taken into consideration. 


\section{Results and Analysis}

\subsection{Numerical Simulation Results Analysis}

It can be concluded that, although fluid has different working conditions in rectangular channel, their change trend are similar. Figure 2 shows the distribution of transverse section temperature field, it is obviously that the temperature in the exit of channel is lower than entry and the temperature surround dimples are lower especially close to dimples, it proves good heat transfer enhancement of dimples. In addition, the windward side of dimples has sparse temperature distribution, heat exchange has lower change and keeps a level, however, the leeward side of dimples has concentrated temperature distribution and has obvious decrease trend of temperature, higher heat exchange rate. With the increase of dimple depth, heat exchange rate is getting stronger. Figure 3 shows the transverse section velocity vector when fluid flows through the channel, we can see the flow rate and direction has changed when fluid pass through dimples, upstream velocity increases and divided when reach downstream air. Besides, adjacent heave force flow field overlying together thus enhances flow strength. It is similar to thread, heave can not only increase heat exchange area, but also can enhance turbulent fluctuation, cut off boundary layer so that enhance turbulent kinetic, in order to achieve good heat transfer enhancement.

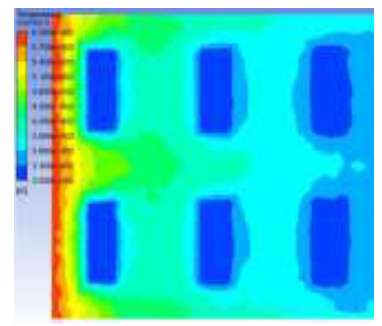

$\mathrm{H}_{\mathrm{d}}=1.6$

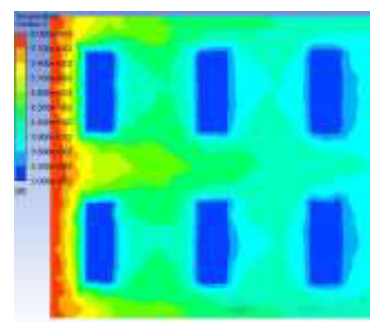

$\mathrm{H}_{\mathrm{d}}=1.3$

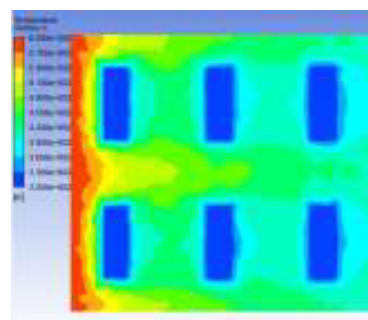

$\mathrm{H}_{\mathrm{d}}=0.8$

Fig. 2. Temperature areas of $H_{d}=0.8 \sim 1.6$

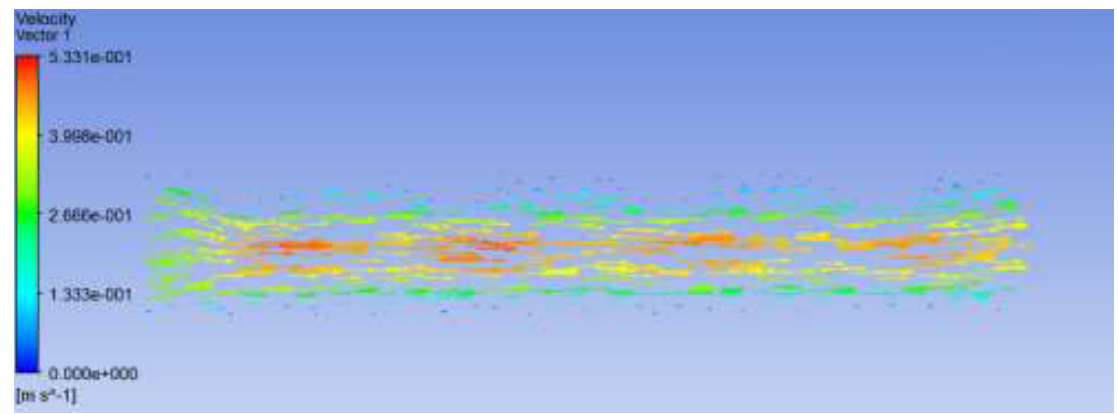

Fig. 3. Velocity vector of $H_{d}=0.8$

\subsection{Data Analysis}

(1) The effect of channel height on dimple heat transfer enhancement. The numerical simulation of model $\mathrm{H}=2 \mathrm{~mm}$, $4 \mathrm{~mm}, 6 \mathrm{~mm}, 8 \mathrm{~mm}$ in $\mathrm{Re}=2000,4000,7000,10000$, the results are shown in figure 4 below. It illustrates that, when Re increased, $\mathrm{Nu}$ increased and $\mathrm{f}$ decreased. When channel height decreased, $\mathrm{Nu}$ and $\mathrm{f}$ increased. It is because when Re is constant, narrow channel leads to higher flow velocity, turbulent increased thus heat transfer is enhanced. The figure 4 
also shows that when Re increased, $\eta$ decreased gradually, the curve of $\eta$ intersected when $\operatorname{Re}=4000$, higher channel height leads to better heat transfer performance. In practical works, according to certain condition, channel height can be increased when $\mathrm{Re}$ is big and decreased channel height when $\mathrm{Re}$ is small so that heat exchange integrated performance can be optimized.

(2) The effect of dimple depth on dimple heat transfer enhancement. The numerical simulation of model $\mathrm{Hd}=0.4 \mathrm{~mm}$, $0.8 \mathrm{~mm}, 1.3 \mathrm{~mm}, 1.6 \mathrm{~mm}$ in $\mathrm{Re}=2000,4000,7000,10000$, the results are shown in figure 5 below. It illustrated that, heat transfer performance is better in dimple channel than in smooth channel, it is because dimple increased the heat exchange area and also breaks the boundary layer thus increased fluid kinetic and turbulent. When $\mathrm{Re}$ increased, $\mathrm{Nu}$ increased and $f$ decreased, as a result of increasing Re caused turbulent enhanced heat transfer performance. When $\mathrm{Re}=$ 4100 , with dimple depth increased, $\eta$ increased. It is because in a small Re condition, the increase gradient of dimple heat transfer is bigger than friction. Above all, $\mathrm{Hd}=0.8 \sim 1.3 \mathrm{~mm}$ has a good heat transfer performance.

(3) The effect of dimple distance on dimple heat transfer enhancement. The numerical simulation of model $\mathrm{S}=4 \mathrm{~mm}$, $9 \mathrm{~mm}, 14 \mathrm{~mm}, 18 \mathrm{~mm}$ in $\mathrm{Re}=2000,4000,7000,10000$, the results are shown in figure 6 below. It illustrated that, with $\mathrm{S}$ decreased, $\mathrm{Nu}$ and $\mathrm{f}$ decreased, it is because when $\mathrm{S}$ went up, dimple concentration decreased, the effect to flow channel will be weaken. When Re increased, $\eta$ decreased gradually, there was a threshold point of four different dimples when $\mathrm{Re}=5200$. Dimple distance has negative effect on heat transfer performance when $\operatorname{Re}<5200$ and good effect when $\mathrm{S}=9 \mathrm{~mm}$ when $\mathrm{Re}>5200$. It illustrated that when Re keeps a low value, the heat exchange caused by dimple concentration is stronger than friction, however in a high Re condition, terrible distribution of dimples may lead to bad result, so a good value of $\mathrm{Re}$ is necessary.
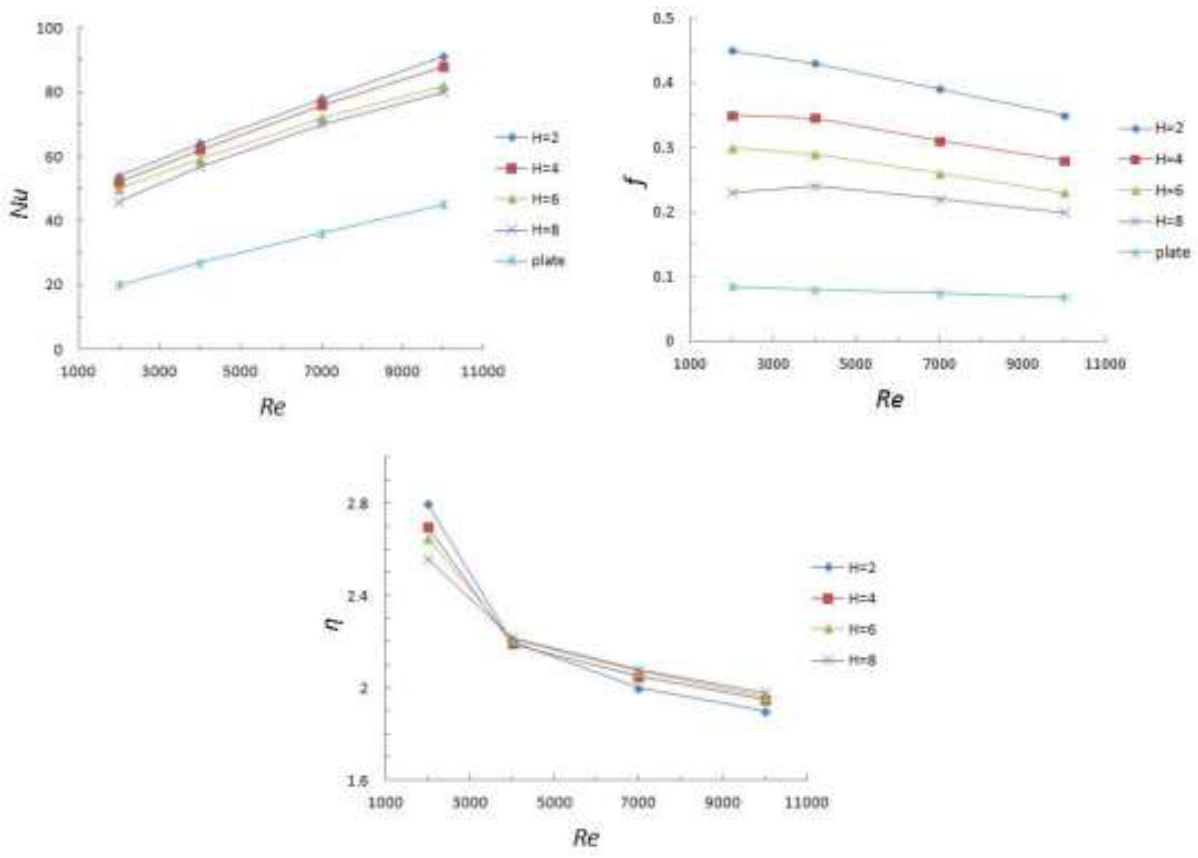

Fig. 4. The trend of $N u, f, \eta$ by changing Re in condition of different $\mathrm{H}$. 

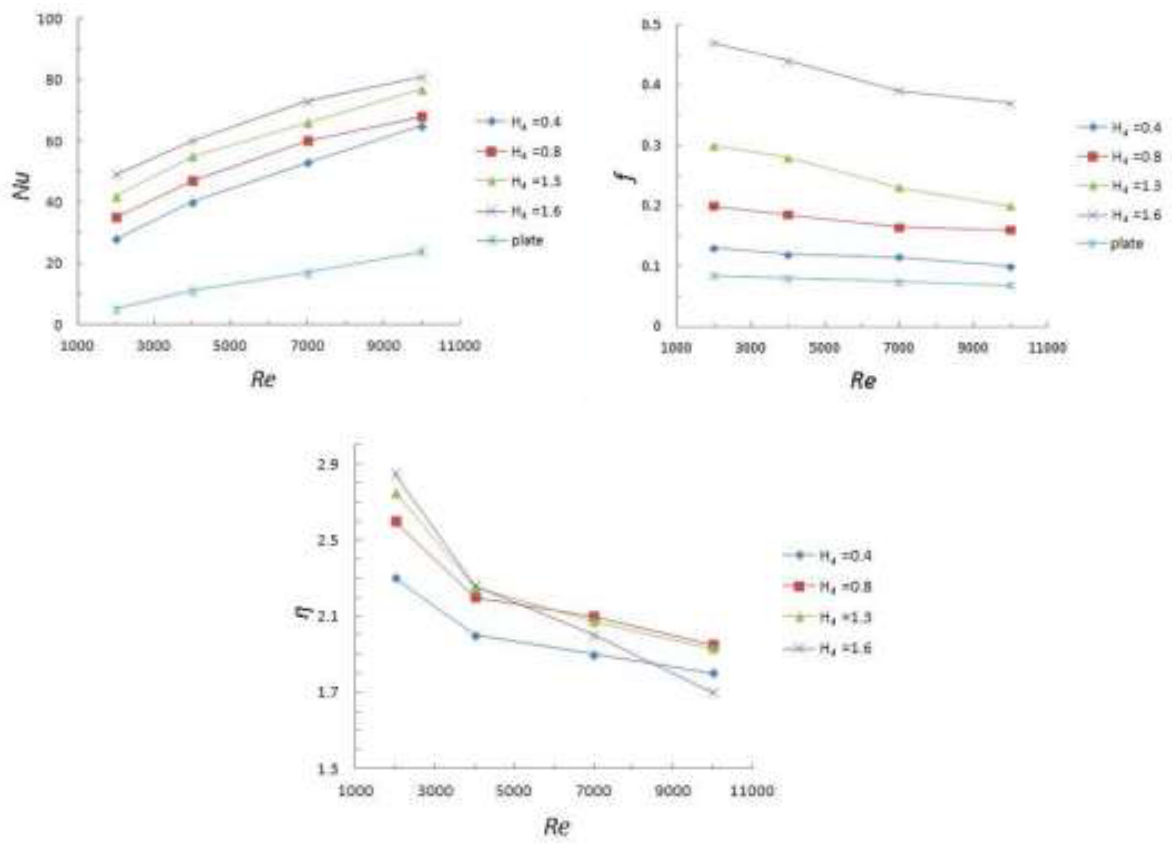

Fig. 5. The trend of $N u, f, \eta$ by changing Re in condition of different $\mathrm{H}_{\mathrm{d}}$.
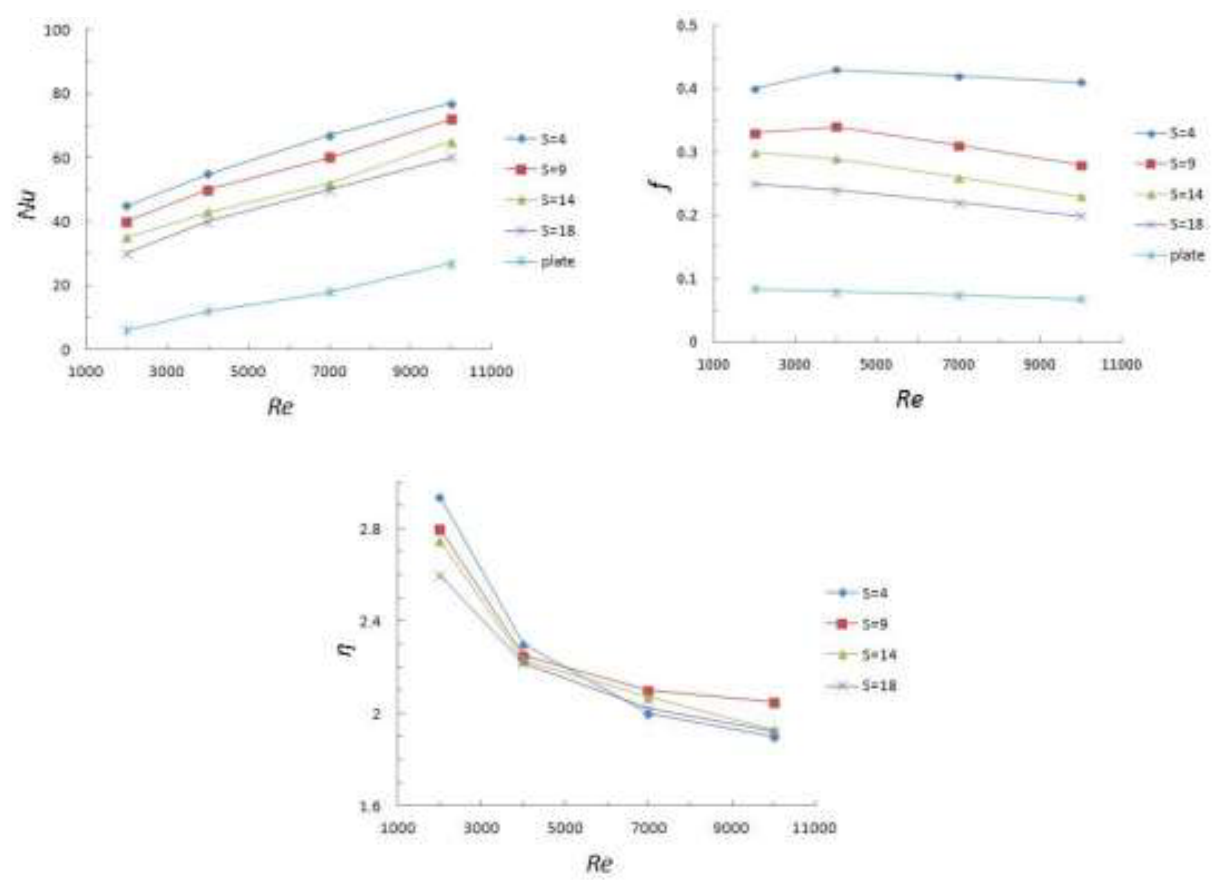

Fig. 6. The trend of $N u, f, \eta$ by changing Re in condition of different $\mathrm{S}$.

\section{Conclusion}

According to the different numerical simulation analysis of heat transfer performance and friction in dimple rectangular channel under different geometry factors conditions, it is concluded that: 
(1)The existence of dimple changed the flow velocity and direction of fluid and broke the internal status.

(2)The existence of dimple increased heat exchange area and broke boundary layer thus increased fluid kinetic and increased the ability of heat exchange.

(3)When Re is between 2000 10000, Nu increased following the increase of Re, $\eta$ decreased when Re increased.

(4)When Re is between 2000 4100, ๆdecreased when H increased, pincreased when Hd increased. When Re is between 5200 10000, ๆhas no obvious trend when other factors changed, however there is an optimized construction that has best heat transfer performance: $\mathrm{H}=8 \mathrm{~mm}, \mathrm{H}_{\mathrm{d}}=0.8 \mathrm{~mm}, \mathrm{~S}=9 \mathrm{~mm}$.

\section{References}

1. G.I. Mahmood, M.L. Hill, D.L. Nelson, et al. Local heat transfer and flow structure on and above a dimpled surface in a channel, ASME J Turbomach, 123,1(2001)

2. V.S. Kesarev, A.P. Kozlov, Convective heat transfer in turbulized flow past a hemispherical Cavity, Heat Transfer Res, 25, 2(1994)

3. V.I. Terekhev, S.V. Kalinina, Y. M. Mshvidobadze, Flow structure and heat transfer on a surface with a unit hole depression, Russ J Eng Thermophys, 5(1999)

4. N. K. Burgess, P. M. Ligrani, Effects of dimple depth on nusselt numbers and friction factors for internal cooling in a channel, ASM E Paper GT, (2004)

5. G.I. Mahmood, M. L Hill, D.L. Nelson, et al., Local heat transfer and flow structure on and above a dimpled surface in a channel, ASM E J.of Turbomachinery, 123,1(2001)

6. Juin Chen, M.S. Hans, G.D. Geoffrey, Heat Transfer Enhancement in Dimpled Tubes, Applied Thermal Engineering, 21 (2001)

7. R.S. Bunker, K.F. Donnellan, Heat transfer and friction factors for flows inside circular tubes with concavity surfaces//ASME Turbo Expo 2003, collocated with the 2003 International Joint Power Generation Conference. American Society of Mechanical Engineers, (2003)

8. S.M. Shin, K.S. Lee, S.D. Park, J.S. Kwak, Measurement of heat transfer coefficient in the dimpled channel: effects of dimple arrangement and channel height, Journal of Mechanical Science and Technology, (2009) 